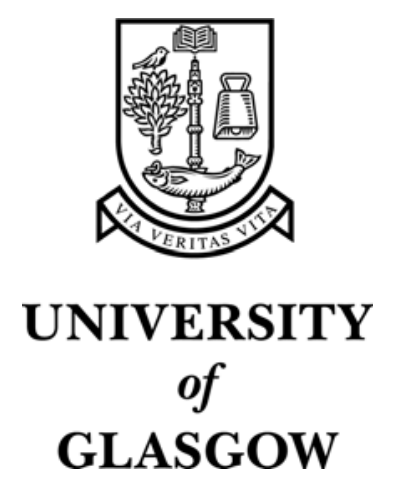

McGee, M.R. and Gray, P.D. and Brewster, S.A. (2001) The effective combination of haptic and auditory textural information. Lecture Notes in Computer Science(2058):pp. 118-126.

http://eprints.gla.ac.uk/3215/ 


\title{
The Effective Combination of Haptic and Auditory Textural Information
}

\author{
Marilyn Rose McGee, Phil Gray, Stephen Brewster
}

\author{
Multimodal Interaction Group \\ Glasgow Interactive Systems Group \\ Department of Computing Science, University of Glasgow, Glasgow, G12 8QQ, UK \\ Email-mcgeemr@dcs.gla.ac.uk \\ Tel - (0141) 3303541 \\ Internet - http://www.dcs.gla.ac.uk/ mcgeemr
}

\begin{abstract}
With the increasing availability and quality of auditory and haptic means of interaction, it is not unusual to incorporate many modalities in interfaces rather than the purely visual. The user can be powerfully affected however when information presented in different modalities are combined to become multimodal. Providing interface designers with the means to implement haptic-audio interfaces might result in adverse effects to interaction unless they are also equipped with structured knowledge on how to select effective combinations of such information. This work introduces 'Integration of Information' as one important dimension of haptic-audio interaction and explores its effects in the context of multimodal texture perception. The range and resolution of available textures through force feedback interaction is a design consideration that might benefit from the addition of audio. This work looks at the effect of combining auditory and haptic textures on people's judgment of the roughness of a virtual surface. The combined haptic-audio percepts will vary in terms of how congruent they are in the information they convey regarding the frequency of bumps or ridges on the virtual surface. Three levels of integration (conflicting, redundant, or complementary) are described and their possible implications discussed in terms of enhancing texture perception with force-feedback devices.
\end{abstract}

\section{Keywords}

Haptic, audio, force-feedback, texture perception, multimodal information processing, intersensory integration.

\section{INTRODUCTION}

\section{Motivations}

Multimodal Interfaces involve the use of multiple human modalities in the interaction (input, output, or both) between the human user and the computer. Haptic-audio interfaces therefore involve the use of both haptic and audio means of interaction (see Table 1. for definitions). In particular, the term haptic-audio interfaces is used here to refer to the communication of certain information to the user through an interface using a combined haptic and audio representation of this information rather than a single modality representation. The advances in both haptic and audio technology have resulted in such haptic-audio interfaces becoming increasingly realistic to implement in a wide range of applications yet we have little organized knowledge on how best to design them. This work contributes to a body of knowledge on how to effectively combine haptic and auditory information.

The way we integrate information from different sensory modalities is complex (Wickens et al, 1983) and can seriously contribute to the quality of interaction in multimodal interfaces. The term 'integration of information' is used to refer to the information processing involved in combining two (or more) different modalities presented together to convey the same piece of information. Two modalities can be combined and the resulting multimodal percept may be a weaker, stronger, or altogether different percept. The effects of combining haptic and audio information must therefore be systematically explored to realize the potential of haptic-audio 
interfaces as well as to avoid creating interfaces that afford poor interaction.

There are specific interaction issues emerging from the increasing use of haptic interfaces, which could potentially be solved using careful addition of audio. One such interaction issue is that of haptically representing texture. In particular, force feedback devices are being used to convey texture by perturbing the user's hand or finger movements kinesthetically rather than cutaneously as with tactile devices (e.g. Lederman, 1999; West and Cutkosky, 1997). This often relies on much larger forces than those typically experienced on the skin during real texture perception (Katz, 1989). We have found in our previous work that such gross textures can perturb the users' movements so much that the ability to stay on the textured surface is adversely affected (Oakely et al 2000).

\section{Goals}

This work discusses and empirically evaluates the dimension of 'Integration of Information' in the specific context of haptic-audio texture perception. The goals of the ongoing work are to: (a) explore the effects of combining haptic and audio information at varying levels of integration and (b) determine the potential benefits of using haptic-audio percepts of texture to overcome the limitations of presenting texture through force feedback alone.

\section{Previous Research}

Within multimodal research, there have been distinct areas of specialized interest emerging. It has become clear from the research that exploring how our sense modalities behave in interaction should allow us to choose appropriate combinations of modalities according to the devices being used, the population of users, the environment, and the nature of the task.

Much of the work to date has focused on coordinating multimodal input for example (e.g. Oviatt, 1997), or the coordination of multimodal output for a specialized population such as visually impaired or physically disabled users (e.g. Mynatt, 1997; Stevens et al, 1997). Less work exists on the systematic study of how the combination of multimodal output of information could be better designed to coincide more closely with human information processing capabilities during multimodal interaction. In addition, little work exists on matching these information-processing capabilities to the nature of the interaction device(s) being used.

Visual displays have dominated interface research in the past but more recently auditory displays have been developed and tested (e.g. Brewster, 1997; Mynatt, 1997). With the lack of touch in interfaces now being strongly challenged, haptic technologies have also emerged at a rapid rate (Srinivasan, 1997). With the visual, auditory, and haptic channels (see Table 1. for definitions) all now technically available, multimodal interfaces can reach wider populations, increase the potential realism of displays, and generally increase the quantity and quality of information we can convey through the interface.

In human sensing and manipulation of everyday objects, the perception of surface texture is fundamental to accurate identification of an object (Katz, 1989). In a virtual world also, haptic texture information can both increase the sense of realism of an object as well as convey informational content regarding what the object is, where it is, what it is for and so on (Jansson et al, 1998).

Textures might be used in human and veterinary virtual medicine to assist in diagnosis of certain conditions. The texture of a tissue might indicate how well scarred tissue is healing for example. Using texture in the visualization of data could allow areas of interest to be 'textured' in the same way as colours are used in graphical visualization. Different textures could indicate different keys on a graph or chart for example. Being able to discriminate between various virtual textures in the textile industry might also prove beneficial. With an increasing number of customers shopping online for a variety of products, being able to convey different textures of objects will become crucial. For a variety of reasons it is desirable to be able to represent textures as effectively as possible in virtual environments.

There has been considerable previous work investigating the perceptual aspects of real surface textures. Lederman et al. (1974) suggest that texture perception is mediated by force cues created by spatial geometry of the surface. It is also possible that surface texture perception uses vibratory cues generated by the repeated and regular stimulation of mechanoreceptive 
afferents as the finger is moved across a surface (Katz, 1989). In fact, it is possible that both kinds of cues are involved, depending on the task to be executed (Weisenberger and Krier, 1997). Far less is known about the perceptual response to virtual surfaces. The physical properties of textures are very complex and are proving difficult to reproduce for virtual textures. For example, is a rough surface characterized by irregular or regular surface elements? What effect does inter-element spacing have on perceived roughness? Representing texture with force feedback devices in particular has proved problematic.

Force feedback devices detect changes in the device's configuration and then use mechanical actuators to apply appropriately calculated forces back to the user. Importantly, the interaction relies on kinesthetic information being conveyed to the user rather than cutaneous information (see table 1). These devices often simulate textures with larger forces than those experienced in real texture perception. In our previous work for example we found that the gross textures implemented perturbed users' movements making it hard for them to stay on a desktop target (Oakley et al., 2000).

\begin{tabular}{|l|l|}
\hline Haptic & Relating to the sense of touch. \\
\hline Kinesthetic & $\begin{array}{l}\text { Meaning the feeling of motion. } \\
\text { Relating to sensations originating in } \\
\text { muscles, tendons and joints. }\end{array}$ \\
\hline Cutaneous & $\begin{array}{l}\text { Pertaining to the skin itself or the } \\
\text { skin as a sense organ. Includes } \\
\text { sensation of pressure, temperature, } \\
\text { and pain. }\end{array}$ \\
\hline Tactile & $\begin{array}{l}\text { Pertaining to the cutaneous sense but } \\
\text { more specifically the sensation of } \\
\text { pressure rather than temperature or } \\
\text { pain. }\end{array}$ \\
\hline Force Feedback & $\begin{array}{l}\text { Relating to the mechanical } \\
\text { production of information sensed by } \\
\text { the human kinesthetic system. }\end{array}$ \\
\hline
\end{tabular}

Table 1: Definitions (Oakley et al., 2000)

It could perhaps be argued that texture is more suitable to production by tactile devices. Despite the early perceptual and physiological arguments for a spatial code to texture, three-dimensional force feedback interfaces are able to simulate surface texture (Weisenberger and Krier, 1997). It is the degree of fidelity and realism achievable with such devices that is of primary interest. The interaction issue then is how to overcome any limitations of using force feedback devices alone to represent texture.
The display of a convincing haptic percept such as texture should not necessarily be limited to the haptic modalities. Audio and visual cues can be associated with a haptic display to contribute to the realism or informational content of the display (Rosenberg, 1994). The current work investigates the conditions under which audio cues do and do not enhance force feedback based texture perception.

\section{CURRENT WORK}

It would be beneficial to know the extent to which we can affect peoples' perception by coupling auditory and haptic percepts in a systematic way. In doing so we can establish ways in which to manipulate what the user will perceive at the interface. In particular, we could use this information to overcome limitations of a device. For instance, the addition of audio information to force feedback virtual surfaces might increase the range and/or resolution of textures available to the designer. Likewise, this information could be used to avoid coupling percepts that result in perceptual or cognitive conflict and which in turn might adversely affect the processing of that information.

In the current work, haptic and auditory textures will be rated by a group of participants to establish how rough each stimuli is in terms of each of the other stimuli. This will result in a set of haptic and audio textures identifiable along the dimension of increasing roughness. These haptic and audio stimuli can then be combined to produce multimodal haptic-audio roughness percepts in the main study. The combined textures will be either congruent or incongruent in terms of the information each modality conveys regarding the number of ridges/bumps on the virtual surface. Resulting multimodal percepts might provide redundant, complementary, or conflicting haptic-audio information. The effects of the different levels of congruency and resulting levels of integration of the information will be discussed.

\section{Device}

The PHANToM 1.0 force feedback device by SensAble Technologies will be used to create the haptic virtual surfaces (see Fig. 1). Force feedback devices have optical sensors that detect changes in the device's configuration. The device then uses mechanical actuators to apply forces back to the user calculated from the positional information and the stored algorithmic models of the objects with which the user is interacting. 
The interaction relies on kinesthetic information being conveyed to the user rather than cutaneous information (see table 1).

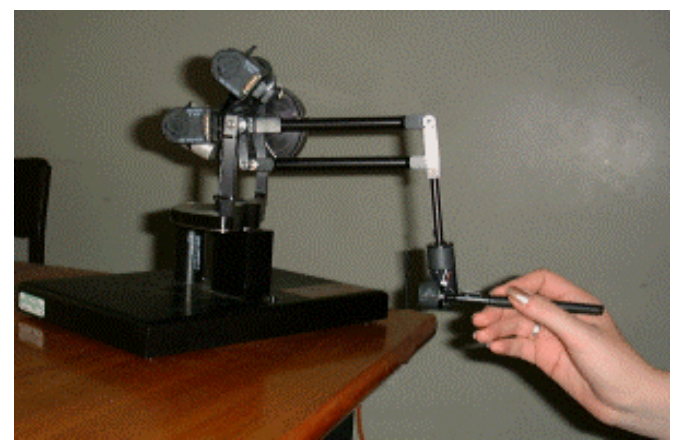

Fig 1: The Phantom 3D force feedback device from SensAble Technologies.

Subjects interact with the device by holding a pen-like stylus attached to a passive gimbal. The user is instructed to scrape the probe of the PHANToM back and forth across the textured area to produce the haptic and/or auditory feedback regarding the roughness of the surface. The stylus switch on the probe of the PHANToM is used to select any response a participant has to make.

\section{Haptic and Auditory Textures \\ Neither haptic nor auditory textures are designed to necessarily model physically accurate or optimum representations of a rough surface. Rather, they are designed to give feedback approximate to that obtained when real textures are explored. In this way, the actual effects of experiencing such feedback multimodally as opposed to unimodally can be explored.}

The haptic textures are generated as sinusoidal gratings on a rectangular patch on the back wall of the workspace. Forces are modeled as a point contact in the $\mathrm{z}$-direction. The resulting profile depends on the amplitude and frequency of the 'wave'. The haptic textures will have a fixed amplitude of $0.5 \mathrm{~mm}$ and frequency (cycles per fixed length of surface) can have one of 6 values - $10,15,20,25,30$, or 35 cycles.

The auditory textures will consist of a sound played to indicate contact with a ridge/bump on the haptic virtual surface. The number of contact sounds can be matched to the number of ridges/bumps experienced haptically (congruent) or provide more or less contact sounds than there are haptic bumps/ridges (incongruent). The exact effect of this congruency/incongruency on the perceived level of roughness of a percept is the subject of investigation.

\section{Manipulating Congruency}

Congruency/Incongruency are determined by the information provided by each modality relating to the number of bumps/ridges encountered on a virtual surface. If the number of contact sounds matches the number of haptic bumps/ridges then they are defined as congruent. Incongruency occurs when the number contact sounds does not match the number of haptic bumps/ridges.

Incongruency however has directionality. Audio information might indicate more or less bumps/ridges than the haptic information. In this case, the incongruency could act to move the level of perceived roughness of a surface up or down the roughness dimension. The direction of incongruency will depend on how frequency of the haptic bumps/ridges, and frequency of contact sounds, unimodally map to level of perceived roughness.

\section{Measuring Perceived Roughness}

Surface roughness is one of texture's most prominent perceptual attributes. The precise physical determinants of roughness however are not exactly clear (e.g. Lederman, 1974). Because there is still debate over the actual parameters that determine roughness, users' perception of virtual roughness (regardless of the underlying physical model) is an increasingly important issue in virtual haptic interaction.

Participants will make a fixed choice response regarding a pair of surfaces. The roughest surface can be on the left, the right, or they can be judged as the same roughness. The proportion of times a surface is judged as rougher than each of the other surfaces can be obtained and the surfaces can then be placed along the roughness dimension.

\section{Task and Procedure}

The haptic-audio surfaces will be presented in pairs as rectangular patches on the back wall of the workspace (see Fig. 2). Participants will be instructed to scrape the probe of the PHANToM back and forth across the stimulus surface to form an impression of how rough the surface seems to them. They will be asked to try to maintain the same speed throughout the experiment. The participant will then be asked to make a judgment regarding their comparison of 
the two surfaces. They make their response by clicking the appropriate button on the screen with the stylus switch on the probe of the PHANTOM.

Clicking the button labeled 'next' will present the next pair of surfaces. When the participant has completed all the trials they will be given a message indicating that they are finished the experiment and a summary file for their responses will automatically be stored for that participant.

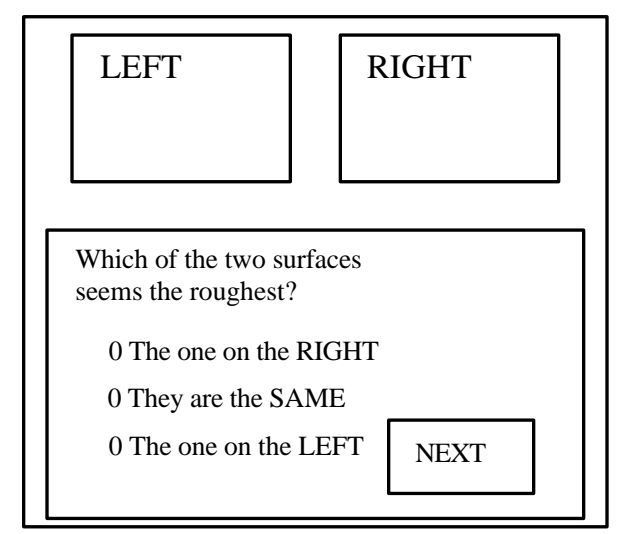

Fig. 2 Diagrammatic view of interface.

\section{HYPOTHESES AND IMPLICATIONS Integration of Information}

Haptic-audio percepts of texture may reduce, increase, or completely alter the informational content of the percept being conveyed multimodally. The exact effects of the hapticaudio coupling will depend on the level at which the information is integrated. The level at which the multimodal information is integrated will depend, in part, on the level of congruency between the haptic and audio stimuli.

Participants will experience congruent and incongruent pairings of haptic and audio textures. The level of integration of these combinations can be conflicting, redundant, or complementary, each of which has the potential to affect perception and resulting interaction in different ways.

H1 - Conflict: If information processed by multiple modalities attempts to convey conflicting information is some way then the resulting multimodal percept may become distorted or completely lost in the process. Alternatively, the judgment of the multimodal percept might change in some unpredictable way.

If the audio stimulus and haptic stimulus are incongruent and conflicting then multimodal (haptic-audio) judgments of roughness will move along the roughness dimension but in the opposite direction predicted by the direction of the incongruency.

H2 - Redundancy: People might process only one modality of information from the many available to them in a multimodal percept. The modality employed may depend on physical/perceptual ability, personal preference, or the nature of the task for example. The actual effects of providing redundant information are somewhat difficult to predict. Redundant information might increase the mental representation of the information. This may in turn lead to increased confidence or reliability of judgments without necessarily altering the content of the information.

If the audio stimulus and haptic stimulus are congruent and redundant then with or without the auditory information, perceptual judgments of a virtual surface will be essentially the same. That is, the unimodal (haptic) and multimodal (haptic-audio) judgments of roughness will be at the same level along the roughness dimension.

H3 - Complementarity: A percept composed of multiple modalities might combine to in fact give more than the sum of the individual parts. That is, two unimodal percepts, when combined, produce some additive effect not possible with either unimodal percept alone. Such complementary pairings of haptic and audio stimuli might act to increase the quality and/or quantity of information available through a haptic-audio interface.

If the audio stimulus and haptic stimulus are incongruent but complementary then multimodal (haptic-audio) judgments of roughness will move along the roughness dimension in the direction predicted by the direction of the incongruency. That is, when an audio and haptic stimulus are combined such that the audio stimulus is more rough than the haptic stimulus then the multimodal judgment of roughness is moved along the roughness dimension in the direction of increasing roughness. Likewise, when an audio stimulus and haptic stimulus are combined such that the audio stimulus is less rough than 
the haptic stimulus then the multimodal judgment of roughness is moved along the roughness dimension in the direction of decreasing roughness.

\section{FUTURE WORK}

Perceptual judgments of the unimodal stimuli are currently being gathered in preparation for combining them to produce the haptic-audio percepts. The next stage of the work will be to combine the haptic and audio textures to produce the congruent and incongruent multimodal percepts. This work will shed light on the ability of audio stimuli to alter the effect of haptic virtual stimuli and the different levels at which the haptic-audio precepts are integrated.

Work is underway to conduct an applied experiment of haptic-audio integration during force feedback texture perception. Veterinary simulation and visualization for the blind are being considered as possible applications areas. Results from the current study will serve to provide predictions regarding the effects of coupling haptic and audio information in a more applied example of force-feedback texture perception. Future work will also include a more in depth exploration of the levels at which we integrate haptic and audio information and how such organised knowledge would aid interface designers in the effective combination of haptic and audio information.

\section{ACKNOWLEDGMENTS}

This research is supported under EPSRC project GR/L79212 and EPSRC studentship 98700418. Thanks also go to the SHEFC REVELATION Project, SensAble Technologies and Virtual Presence Ltd.

\section{REFERENCES}

1. Brewster, S.A. (1997). Using Non-Speech Sound to Overcome Information Overload. Displays, 17, pp. 179-189.

2. Jansson, G., Fanger, J., Konig, H, Billberger, K. (1998), Visually Impaired Person's use of the PHANToM for Information about texture and 3D form of Virtual Objects, Proceedings of the Third PHANToM Users Group Workshop, Cambridge, MA: Massachusetts Institute of Technology.

3. Katz, D. (1989) The World of Touch, (Translated by Krueger, L.E.), Erlbaum, Hillsdale, NJ. Original work published in 1925.
4. Lederman, S.J. (1974). Tactile roughness of grooved surfaces: the touching process and effects of macro- and microsurface structure, Perception and Psychophysics, 16, 2, pp. 385-395.

5. Lederman, S.J., Klatzky, R.L., Hamilton, C.L., Ramsay, G.I. (1999). Perceiving Roughness via a Rigid Probe: Psychophysical Effects of Exploration Speed and Mode of Touch, Haptic e-journal, 1,1, (http://www.haptics-e.org).

6. Mynatt, E.D. (1997) Transforming graphical interfaces into auditory interfaces for blind users. Human-Computer Interaction, 12, PP. 7-45.

7. Oakley, I., McGee, M.R., Brewster, S. \& Gray, P. (2000) Putting the Feel in 'Look and Feel', In Proceedings of ACM CHI 2000, The Hague, ACM Press, Addison-Wesley, pp.415-422.

8. Oviatt, S.L. (1997) Multimodal interactive maps: Designing for human performance. Human -Computer Interaction, 12, pp. 131185.

9. Rosenberg, L.B. (1994), Design of a Virtual Rigid Surface: Haptic/Audio Registration, CHI' 94, April 24-28, pp. 257-258.

10. Srinivasan, M.A. \& Basdogan, C. (1997), Haptics in Virtual Environments: Taxonomy, Research Status, and Challenges, Computers and Graphics, 21, 4, pp. 23-34.

11. Stevens, R.D., Edwards, A.D.N., \& Harling, P.A. (1997). Access to mathematics for visually disabled students through multimodal interaction. Human-Computer Interaction, 12, pp. 47-92.

12. West, A.M. \& Cutkosky, M.R. (1997), Detection of Real and Virtual Fine Surface Features with a Haptic Interface and Stylus, Proceedings of the ASME Dynamic Systems and Control Division, 61, pp. 159-166.

13.Wickens, C.D., Sandry, D.L., Vidulich, M. (1983), Compatibility and Resource Competition between Modalities of Input Central Processing, and Output, Human Factors, 25, 2, pp. 227-248.

14.Weisenberger, J.M., Krier, M.J. (1997). Haptic Perception of Simulated Surface Textures via Vibratory and Force Feedback Displays, Proceedings of the ASME, Dynamic Systems and Control Division, 61, pp.55-60. 